\title{
TV White Space Network Power Allocation Using Hybrid Grey Wolf Optimizer with Firefly Algorithm and Particle Swarm Optimization
}

\author{
$\mathrm{KENNEDY} \mathrm{RONOH}^{1 *}$, GEORGE KAMUCHA ${ }^{2}$ \\ ${ }^{1}$ Department of Computer Science, Technical University of Kenya, Nairobi, KENYA. \\ ${ }^{2}$ Department of Electrical and Information Engineering, University of Nairobi, Nairobi, KENYA
}

Abstract: - TV white spaces (TVWS) can be utilized by Secondary Users (SUs) equipped with cognitive radio functionality on the condition that they do not cause harmful interference to Primary Users (PUs). Optimization of power allocation is necessary when there is a high density of secondary users in a network in order to reduce the level of interference among SUs and to protect PUs against harmful interference. Grey Wolf Optimizer (GWO) is relatively recent population based metaheuristic algorithm that has shown superior performance compared to other population based metaheuristic algorithms. Recent trend has been to hybridize population based metaheuristic algorithms in order to avoid the problem of getting trapped in a local optimum. This paper presents the design and analysis of performance of a hybrid grey wolf optimizer and Firefly Algorithm (FA) with Particle Swarm Optimization operators for optimization of power allocation in TVWS network power allocation as a continuous optimization problem. Matlab was used for simulation. The hybrid of GWO, FA and PSO (HFAGWOPSO) reduces sum power by $81.42 \%$ compared to GWO and improves sum throughput by $16.41 \%$ when compared to GWO. Simulation results also show that the algorithm has better convergence rate.

Key-Words: - TV White Spaces, power allocation, cognitive radio, grey wolf optimizer, firefly algorithm, particle swarm optimization.

Received: February 2, 2021. Revised: June 1, 2021. Accepted: June 12, 2021. Published: June 18, 2021.

\section{Introduction}

Spectrum occupancy assessments conducted in Spain, USA, New Zealand, Singapore and Germany [1] and UK [2] indicate that a significant percentage of spectrum allocated to Primary Users (PUs) is not being fully utilized. Spectrum is considered a scarce resource. The number of devices that need access to spectrum continue to increase and yet the available useful spectrum is limited. Dynamic Spectrum Access (DSA) is currently being seen as one of the remedies to spectrum scarcity and spectrum underutilization [3], [4], [5]. This is because DSA provides an efficient way for spectrum sharing and spectrum management. DSA allows the use of any frequency channel not being used by PUs or any other bands that are not being used such as guard bands[6]. TV white space (TVWS) band has attracted interest among the DSA industrial and research community because of its good propagation characteristics. TVWS is the frequency band that is not being used by TV transmitters in the UHF band [7].

In order to improve Quality of Service (QoS) in a TVWS network and to ensure protection of PUs against any harmful interference, there is need to optimize power allocation. Power allocation in a TVWS network is an NP hard optimization problem. Among other heuristic algorithms, population based metaheuristic algorithms are preferred for NP hard optimization problems [8]. This because such algorithms have better ability for global exploration and local exploitation in searching the solution space in addition to having reasonable time complexity [9]. Despite the advantages of population based metaheuristic algorithms, they can get trapped in a local optimum [10] and this results in premature convergence. Recent trend has been to hybridize evolutionary algorithms so as to overcome the shortcoming by improving either the exploration or exploitation ability or both [10], [11]. 
Grey wolf optimizer is a relatively recent population based metaheuristic algorithm that has shown very good performance [12], [13]. Just like other population based metaheuristic algorithms, it can also suffer premature convergence. The objective of this paper is to present the design and analysis of performance of a hybrid of grey wolf optimizer and firefly algorithm with particle swarm optimization (HFAGWOPSO) operators for power allocation in a TVWS network as a continuous optimization problem. This paper seeks to find out whether hybridizing GWO with FA and PSO will improve the performance of GWO. HFAGWOPSO is further compared with a few other population based metaheuristic algorithms. Simulation results show that compared to grey wolf optimizer (GWO), firefly algorithm (FA), PSO, genetic algorithm (GA) and hybrid FA, GA and PSO (FAGAPSO) [14], HFAGWOPSO achieves the highest sum throughput, lowest sum power and the best convergence rate.

The rest of the paper is organized as follows. Section 2 provides a review of related work. Section 3 provides an overview of relevant is algorithms. Section 4 presents the design of HFAGWOPSO. System model and simulation set up are presented in section 5 and 6 , respectively. Performance evaluation of HFAGWOPSO is discussed in section 7. The paper is concluded in section 8 .

\section{Related Work}

This section presents a review of related work on power allocation in a TVWS network using population based metaheuristic algorithms. In [15], we proposed a firefly algorithm based power allocation algorithm for a TVWS network which makes use of a Geo-location Database (GLDB) and that considers interference constraints at both PU and SUs. In this paper, the performance of HFAGWOPSO is compared to that of both FA and GWO.

We compared the performance of various hybrid FA with GA and PSO algorithms for power allocation in a TVWS network in [14]. Results in the paper showed the performance of FA improves when it is hybridized with PSO and GA. In this paper the performance of HFAGWOPSO is compared to that of hybrid FA, GA and PSO (FAGAPSO) as well as pure FA, PSO and GA.

Our previous work in [5] presents the use of modified FA for joint power and spectrum allocation for a TVWS network. FA is modified to solve a continuous-discrete optimization problem. In this paper, only power allocation is considered.

\section{Overview of Relevant Algorithms}

This section provides a brief overview of GWO, FA, GA and PSO algorithms.

\subsection{Grey Wolf Optimizer}

GWO is a population based metaheuristic algorithm that is derived from the social behavior of grey wolves that prefer to live in a pack made up of 5-12 wolves [12], [16]. Grey wolves have a strict social hierarchy. The wolves at the top of the hierarchy are called alphas. The second, third and fourth in the hierarchy are beta, delta and omega, respectively. The alpha makes decisions such as hunting, where to sleep and wake up time. The rest of the wolves have to follow the decision made by the alpha. The beta wolf assists the alpha in the decision making and is the one to take over in case the alpha dies or ages. The beta follows the decision of the alpha but gives instructions to lower ranked wolves. Sentinels, scouts, elders and caretakers all fall into the category of delta wolves. The omegas are the lowest in the ranking and are the last to eat. Omega wolves take instructions from all wolves. A wolf that is not an alpha, beta or omega is called a delta. They follow instructions from alpha and beta but dominate the omega.

Group hunting is another social behavior of grey wolves. Grey wolves will first of all track, chase and approach the prey. After that they will, pursue, encircle and harass the prey until it stops moving. The final phase of the hunting is for the wolves to make an attack towards the prey.

The GWO algorithm models the two social behaviors of wolves of social hierarchy and group hunting. Each wolf in the pack represents a potential solution to the optimization problem. The fittest solution is called the alpha $(\alpha)$. The second and third best solutions are called beta $(\beta)$ and delta $(\delta)$, respectively. The rest of the candidate solutions are assumed to be omega $(\omega)$. The hunting is led by $\alpha, \beta$ and $\delta$. $\omega$ follow these three candidates. Equation (1) is used mathematically model the encircling behaviour of wolves.

$$
\vec{X}(t+1)=\vec{X}_{p}(t)+\vec{A} \cdot \vec{D}
$$

where $D$ is as defined in equation (2), $t$ is the iteration number, $\vec{A}$ and $\vec{C}$ are coefficient vectors as defined in equations (3) and (4), $\overrightarrow{X_{p}}$ is the position of prey, and $\vec{X}$ is the position of grey wolf.

$$
\begin{gathered}
\vec{D}=\mid \vec{C} \cdot \vec{X}_{p}(t)-\vec{X}(t), \\
\vec{A}=2 a \cdot \overrightarrow{r_{1}}-a,
\end{gathered}
$$




$$
\vec{C}=2 \overrightarrow{r_{2}},
$$

where $a$ is decreased linearly from 2 to 0 over the course of iterations, $r_{1}$ and $r_{2}$ are random vectors in $[0,1]$. In order to replicate the hunting behaviour of grey wolves, the alpha, beta and delta are assumed to have better knowledge about the potential position of prey. All the other wolves will then have to update their positions of their search agents according to position of the best search agents (alpha, beta and delta). The position of wolves are updated according to equation (5).

$$
\vec{X}(t+1)=\frac{\vec{X}_{1}+\vec{X}_{2}+\vec{X}_{3}}{3}
$$

where $\vec{X}_{1}, \vec{X}_{2}$ and $\vec{X}_{3}$ are defined in equations (6), (7) and (8).

$$
\begin{aligned}
& \vec{X}_{1}=\vec{X}_{\alpha}-\vec{A}_{1} \cdot\left(\vec{D}_{\alpha}\right), \\
& \vec{X}_{2}=\vec{X}_{\beta}-\vec{A}_{2} \cdot\left(\vec{D}_{\beta}\right), \\
& \vec{X}_{3}=\vec{X}_{\delta}-\vec{A}_{3} \cdot\left(\vec{D}_{\delta}\right) .
\end{aligned}
$$

where $\vec{X}_{\alpha}, \vec{X}_{\beta}$ and $\vec{X}_{\delta}$ are the positions of the first best three solutions, $\vec{A}_{1}, \vec{A}_{2}$ and $\vec{A}_{3}$ are defined in equations (6), (7) and (8) and $\vec{D}_{\alpha}, \vec{D}_{\beta}$, and $\vec{D}_{\delta}$ are defined in equations (9), (10) and (11).

$$
\begin{aligned}
& \vec{D}_{\alpha}=\left|\vec{C}_{1} \cdot \vec{X}_{\alpha}-\vec{X}\right|, \\
& \vec{D}_{\beta}=\left|\vec{C}_{2} \cdot \vec{X}_{\beta}-\vec{X}\right|, \\
& \vec{D}_{\delta}=\left|\vec{C}_{3} \cdot \vec{X}_{\delta}-\vec{X}\right|,
\end{aligned}
$$

where $\vec{C}_{1}, \vec{C}_{2}$ and $\vec{C}_{3}$ are defined in equation (4). Parameter $a$, that contols the balance between exploration and exploitation, is updated according to (12).

$$
a=2-t \frac{2}{\text { MaxIter }}
$$

where $t$ is the iteration number and MaxIter is the maximum number of iterations. Algorithm 1 represents the pseudocode for GWO algorithm.

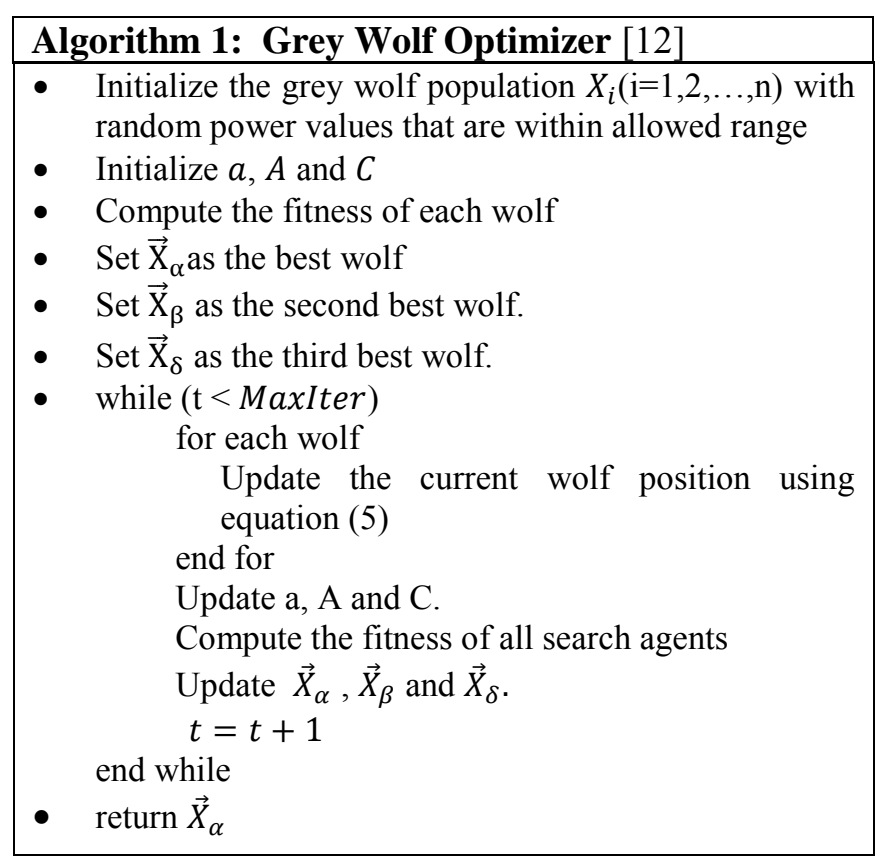

\subsection{Firefly Algorithm}

FA mimics the behavior of fireflies. Firefly is an insect that flash to either attract a mate or potential prey [17]. Flashing may also serve as a warning mechanism. The flashing of a firefly is rhythmic. For female fireflies, the attractiveness of male fireflies depends on its brightness. The light intensity has an inverse relationship with distance.

Light intensity reduces as distance increases according to this formula: $I \propto \frac{1}{r^{2}}$. Fireflies, therefore, are visible within a limited distance. The objective function of an optimization problem can be associated with the flashing. The light intensity is determined by brightness $I$ which is associated with an objective function value. In an optimization problem, each firefly represents a potential solution to the optimization problem. Variation of attractiveness with distance is given by:

$$
\beta=\beta_{o} e^{-\gamma r^{2}}
$$

where the term $\beta$ denotes to firefly light intensity, $r$ is the distance between two fireflies and $\gamma$ is the light absorption co-efficient. For any two flashing fireflies, a firefly with less brightness will move towards a brighter one as per to equation (14).

$$
x_{i}^{t+1}=x_{i}^{t}+\beta_{o} e^{-\gamma r_{i j}^{2}}\left(x_{j}^{t}-x_{i}^{t}\right)+\alpha_{t} \epsilon_{t}^{i},
$$

where the terms $x_{i}$ and $x_{j}$ are the locations of fireflies $i$ and $j, \alpha$ is a randomization parameter and the term $\epsilon_{t}^{i}$ is a vector of random numbers. The first term stands for attractiveness while the second term stands for randomization. The symbol $t$ is the 
iteration number. The distance between fireflies, $r_{i j}$, is computed according to equation (15):

$$
r_{i j}=\sqrt{\left(x_{i, t}-x_{j, t}\right)^{2}} .
$$

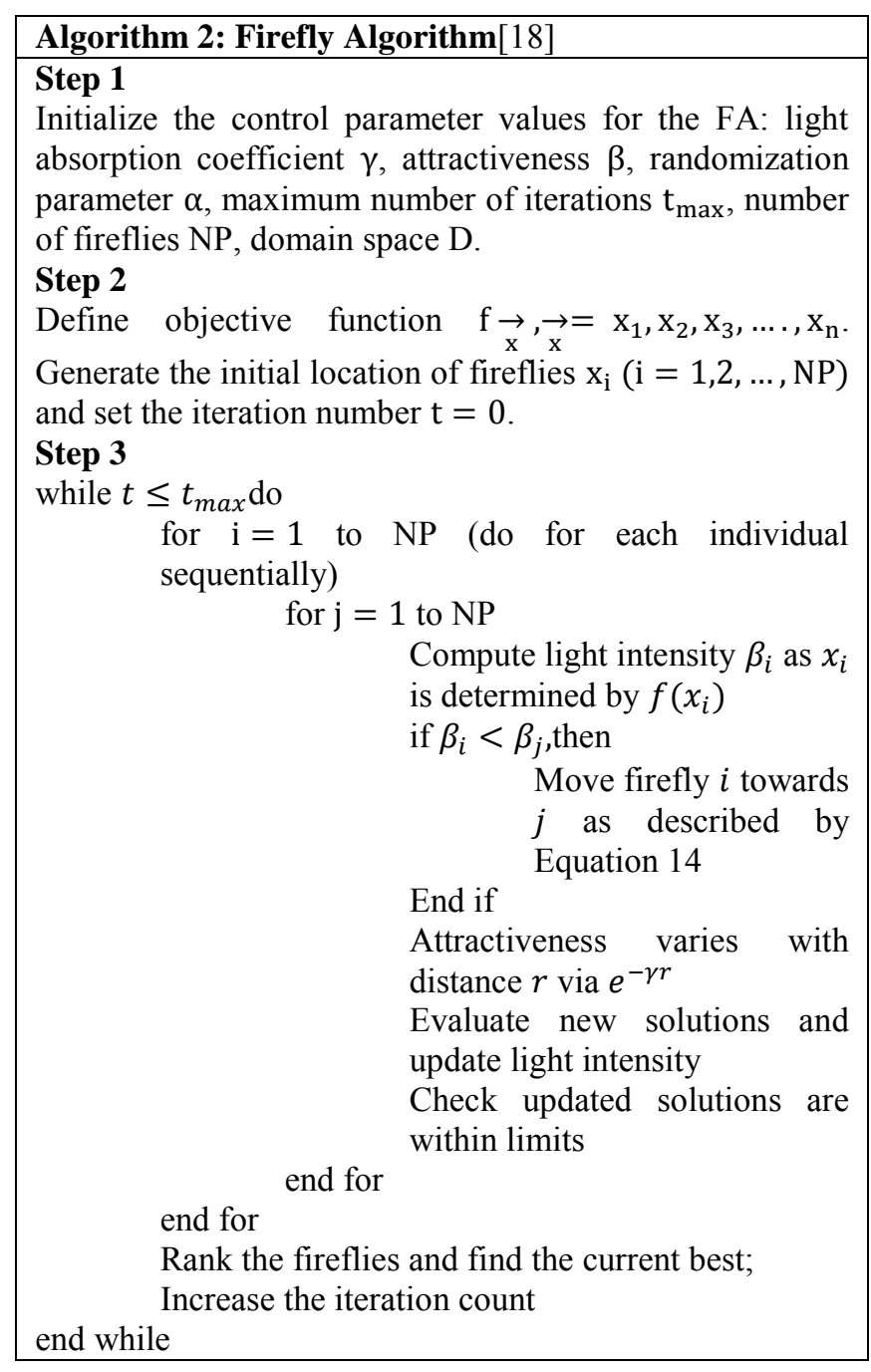

\subsection{Particle Swarm Optimization Algorithm}

PSO is inspired by a flock of birds flying towards a destination. Each candidate solution is referred to as a particle. Each particle represents a bird in the flock. Unlike GA, no new birds/particles are generated. The existing particles are improved iteratively. The birds adjust their social behavior as they move towards the destination. Birds communicate as they fly. As they communicate they identify the bird which is in the best position and then they move towards it at a certain velocity. PSO combines both local search and global search. Local search is represented by each bird learning from their own experience. Global search is represented by each bird learning from the experience of others. PSO starts by generating a set of particles with a random solutions in the to the optimization problem.
The fitness of each particle is then evaluated. Each particle looks at three parameters: its current position $X_{i}$, its current best position $P_{i}$ and associated objective function value $P_{b e s t}$, and its flying velocity $V_{i}$. At every iteration $X_{i}$ and associated objective function value $P_{b e s t}$ is updated if there is an improvement in $P_{i}$. The best particle, $P_{\text {best }}$, is also determined at every iteration. The global best particle $P_{g}$ and associated objective function value $g_{\text {best }}$ is also updated if the current $P_{\text {best }}$ is better than $g_{\text {best }}$ at every iteration. At every iteration also, each particle flies towards $P_{i}$ and $P_{g}$ at a certain velocity. Each particle updates its current velocity, $V_{i}$, according to the equation (16):

New $V_{i}=\omega \times$ current $V_{i}+c_{1} \times \operatorname{rand}() \times\left(P_{i}-\right.$ $\left.X_{i}\right)+c_{2} \times \operatorname{rand}() \times\left(P_{g}-X_{i}\right)$,

where $c_{1}$ and $c_{2}$ are two positive constants and rand() is a random function. The term $\omega$ plays the role of balancing local search and global search. With the new current velocity, the position of the particle is then updated according to the equation (17):

$$
\begin{array}{r}
\text { New position } X_{i}=\text { current position } X_{i}+ \\
\text { New } V_{i}, \quad \text { (17) } \\
V_{\text {min }} \geq V_{i} \geq-V_{\text {max }},
\end{array}
$$

where $V_{\max }$ is the maximum particle velocity and $V_{\min }$ is the minimum particle velocity.

PSO has been applied for power allocation in a $\mathrm{CRN}$ in [19]. In the proposed algorithm, the objective is to maximize signal to interference noise ratio (SINR) for all SUs. Each particle $\left(X_{i}\right)$, represents a potential solution to the problem of finding optimal power and spectrum allocation to all SUs. Initially SUs are assigned power randomly. The objective function used is minimization of minimum SINR violation. At each iteration the best power vector for each particle $\left(P_{i}\right)$ and global best power vector $\left(P_{g}\right)$ are updated if there is an improvement. At every iteration, $X_{i}$ will then moves towards $\left(P_{i}\right)$ and $\left(P_{g}\right)$ at a certain velocity. After a fixed number of iterations, $P_{g}$ will be selected as the optimal solution to the problem of power assignment. 


\subsection{Hybrid Firefly and Particle Swarm Optimization Algorithms}

Arunachalam et. al. [20] proposed a hybrid FA and PSO for problem of combined economic and emission dispatch including valve point effect. In the proposed algorithm, there is no modification to firefly algorithm but the initial solution is obtained from PSO. The authors argue that quality of the final solution of FA depends on the initial solution. Simulation results show that hybrid the algorithm performs better than both PSO and FA.

Kora P. and Krishna K. [21] also proposed a hybrid FA and PSO algorithm for detection of bundle branch block. The hybrid algorithm makes use of PSO concepts and parameters. The concepts of personal best and global best which are absent in FA are introduced. All the steps in FA remain the same with that of the proposed algorithm except that equation (2.2) of the FA that represents firefly movement is changed to incorporate the idea of personal best and global best. In the proposed algorithm, each firefly movement involves a move towards the local personal best $\left(P_{i}\right)$ and global best $\left(P_{g}\right)$.

$x_{i}^{t+1}$

$=x_{i}^{t}+c_{1} e^{-\gamma r_{i j}^{2}}\left(p_{i}-x_{i}^{t}\right)+c_{2} e^{-\gamma r_{i j}^{2}}\left(p_{g}-x_{i}^{t}\right)$

$+\alpha_{t} \epsilon_{t}^{i}$.

\section{Hybridizing Grey wolf optimizer with Firefly Algorithm and Particle Swarm Optimization}

Grey wolf optimizer searches the solution space according to the position of alpha, beta and delta that are assumed to know the position of prey. In GWO, the term $a$ in equation (3) is decreased linearly from 2 to 0 over the course of iterations. At the start, $\vec{A}$ (equation 3 ) has values greater than 1 or less than -1 so that there is exploration through divergence from prey position (approximated by alpha, beta and delta). As a reduces over the course of iterations, divergence from alpha, beta and delta reduces and hence exploration of the solution space becomes limited. Although the values of $r_{1}$ and $r_{2}$ remain as random vectors in $[0,1]$ throughout the course of iterations, their values do not significantly influence exploration.

Grey wolf optimizer is hybridized FA with PSO operators in this paper so as to enhance exploration. This is illustrated in Algorithm 3. Firefly movement using PSO operators is added to the original GWO algorithm in step 2.5 . Firefly movement will add an extra exploration or divergence term to GWO in order prevent premature convergence. Firefly movement using PSO operators is made use of because it was found to enhance the performance of firefly algorithm in [14].

Algorithm 3: Hybrid Grey Wolf Optimizer and Firefly Algorithm with PSO Operators

\section{Step 1: Initialization}

- 1.1 Initialize the grey wolf population $X_{i}(\mathrm{i}=1,2, \ldots, \mathrm{n})$ with random power values that are within allowed range

- $\quad 1.2$ Initialize $a, A$ and $C$

- $\quad 1.3$ Compute the fitness of each wolf

- $\quad 1.4$ Set $\vec{X}_{\alpha}$ as the best wolf

- $\quad 1.5$ Set $\vec{X}_{\beta}$ as the second best wolf.

- $\quad$ 1.6 Set $\vec{X}_{\delta}$ as the third best wolf.

Step 2

- $\quad$ while $(\mathrm{t}<$ MaxIter $)$

2.1 for each wolf Update the current wolf position using equation (5)

end for

2.2 Update a, A and C.

2.3 Compute the fitness of all search agents

2.4 Update $\vec{X}_{\alpha}, \vec{X}_{\beta}$ and $\vec{X}_{\delta}$.

2.5 for each wolf

$$
\text { if } \beta_{\mathrm{i}}<\beta_{\mathrm{j}} \text {, then }
$$
Move firefly $i$ towards $j$ as described by Equation 16 End if

End for

$$
\text { 2.6 } t=t+1
$$

Step 3

end while

- $\quad$ return $\vec{X}_{\alpha}$

\section{System Model}

The optimization problem to be considered is about power allocation optimization described in our previous paper in [15]. Network shown in Figure 1 is considered. One TV receiver put near the border of the protection zone. Of all the TV receivers in the protection zone, a TV receiver at that location is much more vulnerable to interference because it receives the lower transmit power from TV tower compared to other TV receivers. A TV receiver at that location also receives the highest interference from SUs because it is nearest to the secondary cell. Protection ratio at the TV receiver should not fall below the required protection ratio threshold. The network consists of $M$ channels and $N$ SUs.

The optimization problem is defined as follows [15]: 


\section{Problem 1}

$p^{*}=\arg \min \varnothing(p)$

subject to $C: p_{\min } \leq p_{i} \leq p_{\max }$.

where

$$
\begin{aligned}
& \emptyset(p)=\varphi(p)+c_{s} \sum_{i=1}^{N} \max \left[0, g_{i}^{s}\right]^{2} \\
& +c_{p} \max \left[0, g_{i}^{p}\right]^{2} .
\end{aligned}
$$

The optimization problem in Problem 1 is about minimization of sum power and interference threshold violations at SUs and at the PU. In equation (20), the first term, $\varphi(p)$, represents the sum power of all SUs, the second term $\left(c_{S} \sum_{i=1}^{N} \max \left[0, g_{i}^{S}\right]^{2}\right) \quad$ represents interference threshold violation for SUs while the third term represents interference threshold violation for PU. The terms $g_{i}^{s}$ and $g_{i}^{p}$ refer to SINR threshold for SU and PU, respectively. $c_{s}$ and $c_{p}$ are penalty factors for SU interference threshold violation and PU interference threshold violation, respectively.

TVWS power allocation optimization Problem 1 is then solved using Algorithm 4. Each wolf in a prey hunting pack in GWO algorithm represents a potential solution to power allocation in a TVWS network.

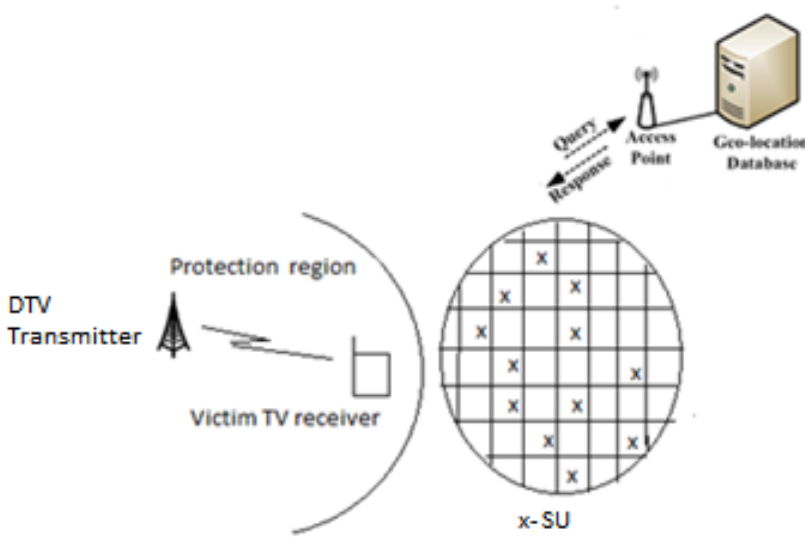

Figure 1: Interference scenario

\section{Simulation Set Up}

Simulation parameters are laid out in Table I. Matlab R2016a was used for simulation. Matlab is selected because it has diverse mathematical functions. SUs $(\mathrm{N}=1000)$ are randomly distributed across an area of $1 \mathrm{~km}^{2}$. Fig. 2 illustrates the network diagram created in Matlab. The channels to be considered are the ones in Nairobi central business district shown in Fig.3.

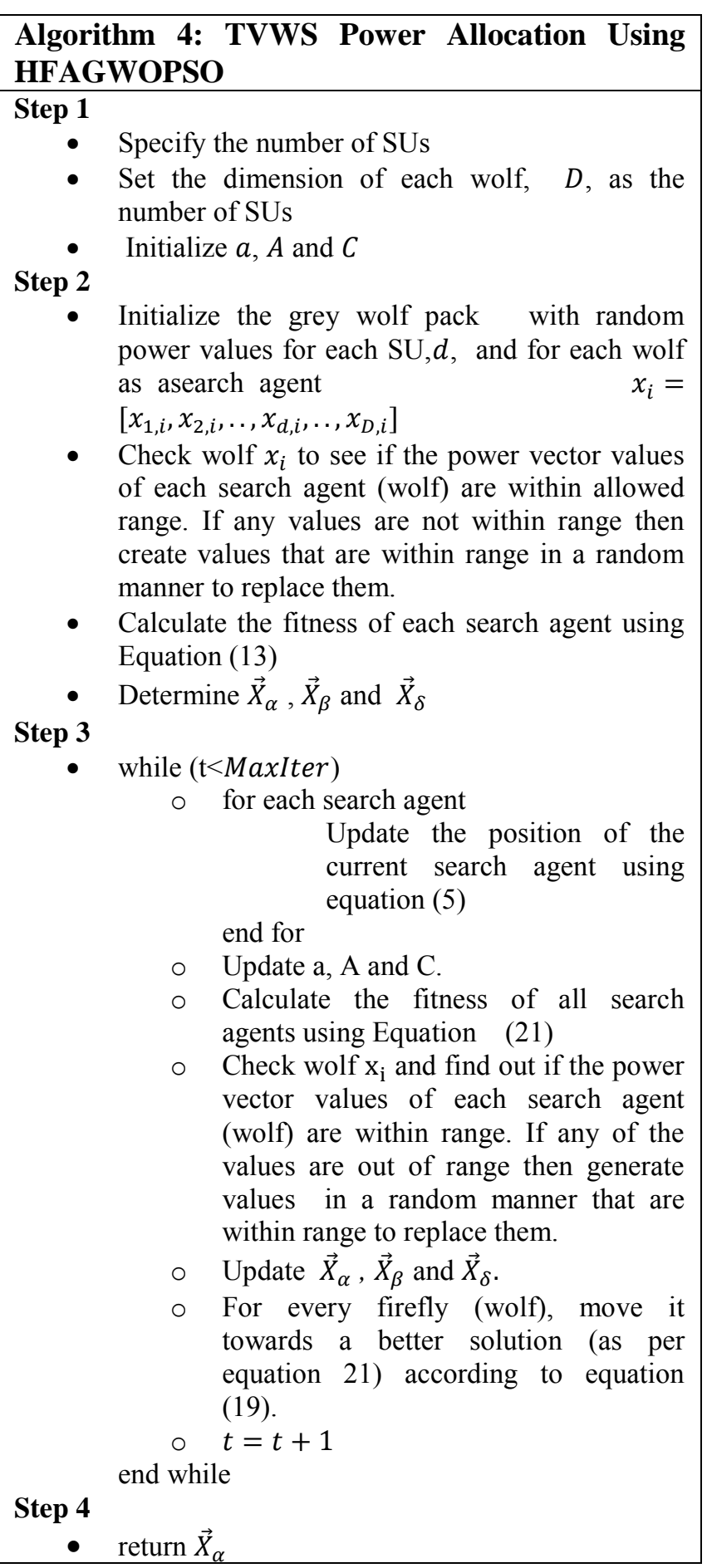

SUs are distributed in a random manner all through the 10 channels $(M=10)$. The free space path loss model was used to model path loss [22]:

$$
P L(d)=20 \log (d)+20 \log (f)-147.55,
$$

where $d$ is the distance from sending antenna to receiving antenna in meters and $f$ is the frequency of the device. Algorithm 2 is then used to assign power to SUs. 
Table I: Simulation Parameters

\begin{tabular}{|c|l|l|}
\hline Parameter & Value & Description \\
\hline$b_{m}$ & $6 \mathrm{MHz}$ & TV channel bandwidth \\
\hline$f_{a}$ & $650 \mathrm{MHz}$ & DTV signal centre frequency \\
\hline$P_{\mathrm{TV}}$ & $-70.6 \mathrm{dBm}$ & $\begin{array}{l}\text { Received DTV signal power } \\
\text { at TV receiver }\end{array}$ \\
\hline$\delta_{n}^{2}$ & $-102 \mathrm{dBm}$ & Noise power \\
\hline$\omega_{o}$ & $23 \mathrm{~dB}$ & $\begin{array}{l}\text { SINR threshold of } \\
\text { receiver }\end{array}$ \\
\hline$\rho_{o}$ & $7 \mathrm{~dB}$ & SINR threshold of SU \\
\hline$P^{B S}$ & $\begin{array}{l}36 \\
(4 \mathrm{~W})\end{array}$ & Base station transmit power \\
\hline$p_{m a x}$ & $30 \mathrm{dBm}$ & $\begin{array}{l}\text { Maximum transmit power of } \\
\text { SU }\end{array}$ \\
\hline$G_{S U}$ & $10 \mathrm{~dB}$ & Antenna gain of SU \\
\hline$G_{P U}$ & $10 \mathrm{~dB}$ & Antenna gain of PU \\
\hline$G_{B S}$ & $10 \mathrm{~dB}$ & Antenna gain of access point \\
\hline
\end{tabular}

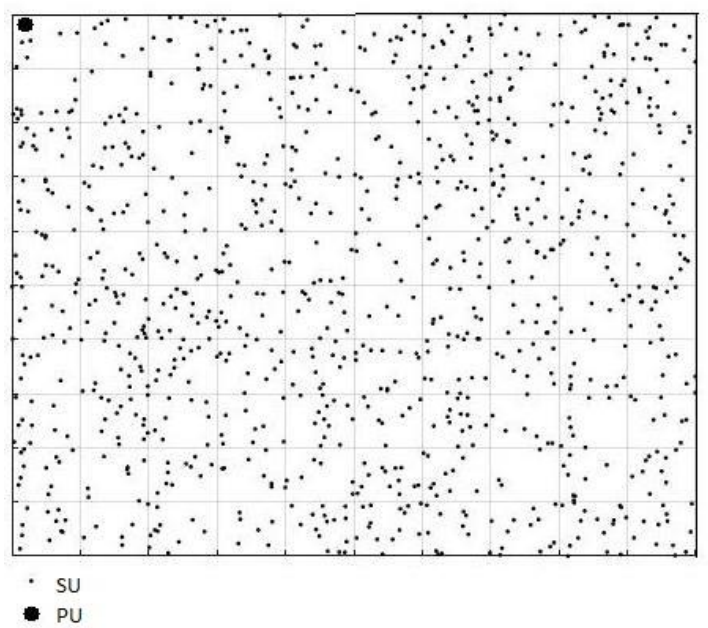

Figure 2: Network Diagram

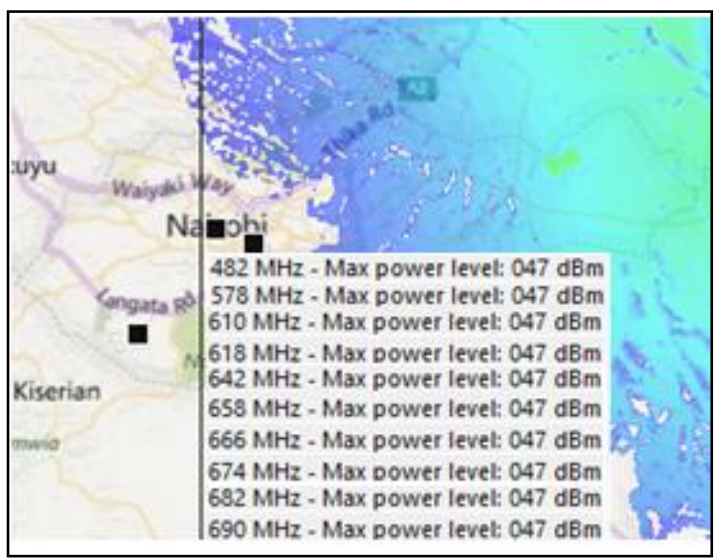

Figure 3: Network diagram

FA parameters used: number of fireflies $N P=50$, $\beta_{o}=1, \alpha=30, \gamma=10$. Parameters used for GWO: $a$ starts with 2. PSO parameters used: inertia weights: $\quad w_{\max }=4$ and $w_{\min }=2$, number of particles $=50$, social parameter $c_{1}=2$ and cognitive parameter $c_{2}=2$. GA parameters used : number of chromosomes $=50$, selection rate $=0.5$ and mutation rate $=0.8$. Parameters used for FA are as follows: $\beta_{o}=1, \alpha=30, \gamma=10$. The number of iterations used is 500. Other parameters used are outlined in Table I.

\section{Performance Evaluation}

In this section, performance of HFAGWOPSO for power allocation in a TVWS network is compared with grey wolf optimizer (GWO), particle swarm optimization (PSO), genetic algorithm (GA) and firefly algorithm (FA). The performance metrics used are sum power, sum throughput, percentage of SUs less than threshold, objective function value and running time.

\subsection{Sum Power}

Table II shows comparison of the HFAGWOPSO with the rest of the algorithms in terms of sum power in the network. The results show that the HFAGWOPSO achieves the lowest sum power. HFAGWOPSO reduces sum power by $81.42 \%$, $99.91 \%, 99.90 \%, 99.91 \%$ and $99.16 \%$ compared to GWO, GA, PSO, FA and FAGAPSO, respectively. This is because of the better power allocation achieved by the algorithm.

Table II: Comparison of Sum Power

\begin{tabular}{|l|l|l|}
\hline Algorithm & $\begin{array}{l}\text { Sum } \\
\text { Power } \\
\text { (Watts) }\end{array}$ & $\begin{array}{l}\text { Percentage } \\
\text { Reduction }\end{array}$ \\
\hline HFAGWOPSO & 1.68 & \\
\hline GWO & 9.04 & $81.42 \%$ \\
\hline GA & 1824 & $99.91 \%$ \\
\hline PSO & 1751 & $99.90 \%$ \\
\hline FA & 1789 & $99.91 \%$ \\
\hline FAGAPSO & 199 & $99.16 \%$ \\
\hline
\end{tabular}

\subsection{Sum Throughput}

Table III shows performance comparison of the HFAGWOPSO with the rest of the algorithms in terms of sum throughput in the network. HFAGWOPSO improves sum throughput by $16.41 \%, 151.70 \%, 150.06 \%, 153.17 \%$ and $60.20 \%$ when compared to GWO, GA, PSO, FA and FAGAPSO, respectively. This is because of the improved power allocation that minimizes interference in the network. According to Shannon channel capacity theorem, reduction in interference improves throughput. 
Table III: Comparison of Sum Throughput

\begin{tabular}{|l|l|l|}
\hline Algorithm & $\begin{array}{l}\text { Sum } \\
\text { Throughput } \\
\text { (Gbps) }\end{array}$ & $\begin{array}{l}\text { Percentage } \\
\text { Increase }\end{array}$ \\
\hline HFAGWOPSO & 65.04 & \\
\hline GWO & 55.87 & $16.41 \%$ \\
\hline GA & 25.84 & $151.70 \%$ \\
\hline PSO & 26.01 & $150.06 \%$ \\
\hline FA & 25.69 & $153.17 \%$ \\
\hline FAGAPSO & 40.6 & $60.20 \%$ \\
\hline
\end{tabular}

\subsection{Percentage of SUs less than SU SINR Threshold}

Table IV shows performance comparison of HFAGWOPSO with the rest of the algorithms in terms of percentage of SUs with SU SINR less than required threshold of $7 \mathrm{~dB}$ in the network. The results show that the GWO achieves the lowest percentage of SUs with SU SINR below threshold. This is because of the improved power allocation that minimizes interference in the network.

Table IV: Comparison of Percentage of SUs Less Than SU SINR Threshold

\begin{tabular}{|l|l|}
\hline Algorithm & $\begin{array}{l}\text { Percentage of SUs } \\
\text { less than SU SINR } \\
\text { Threshold }\end{array}$ \\
\hline HFAGWOPSO & $0.76 \%$ \\
\hline GWO & $1.5 \%$ \\
\hline GA & $15.84 \%$ \\
\hline PSO & $15.66 \%$ \\
\hline FA & $16.54 \%$ \\
\hline FAGAPSO & $5.04 \%$ \\
\hline
\end{tabular}

\subsection{Objective Function Value}

Table V shows comparison HFAGWOPSO with the rest of the algorithms in terms of achieved objective function value. The results show that HFAGWOPSO achieves the best (lowest) objective function value represented by equation (20).

Table V: Comparison of Objective Function Values

\begin{tabular}{|l|l|l|}
\hline Algorithm & $\begin{array}{l}\text { Objective } \\
\text { Function } \\
\text { Value }\end{array}$ & $\begin{array}{l}\text { Percentage } \\
\text { Reduction }\end{array}$ \\
\hline HFAGWOPSO & 2438 & \\
\hline GWO & 2510 & $2.87 \%$ \\
\hline GA & 20600 & $88.17 \%$ \\
\hline PSO & 17318 & $85.92 \%$ \\
\hline FA & 20312 & $88.00 \%$ \\
\hline FAGAPSO & 4415 & $81.09 \%$ \\
\hline
\end{tabular}

\subsection{Rate of Convergence}

Convergence curve for the algorithms under consideration for 300 iterations are shown in Figures 4 and 5. Figure 4 shows a comparison of all the algorithms. Figure 5 shows a zoomed in comparison of convergence curves for HFAGWOPSO, GWO and FAGAPSO.

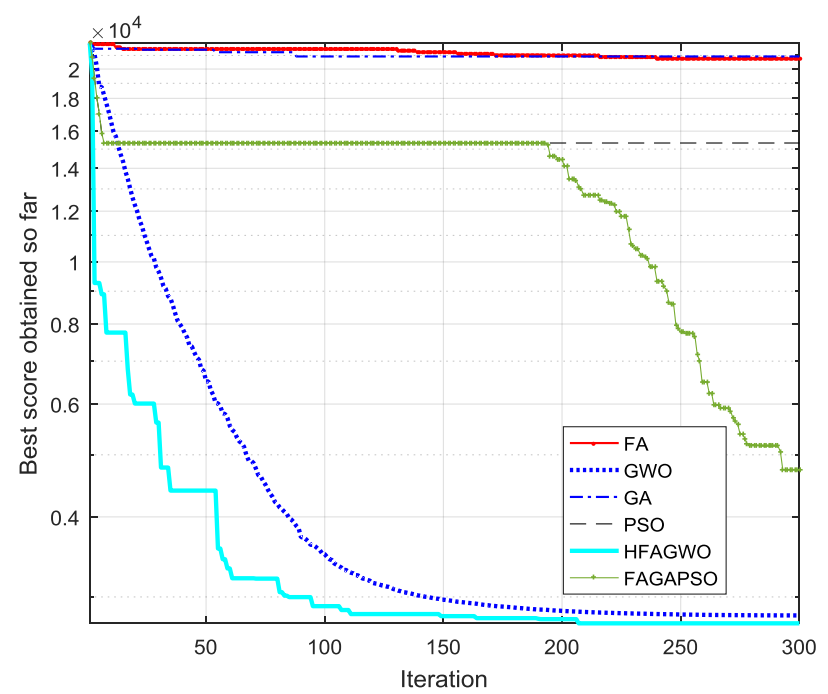

Figure 4: Comparison of Convergence Curve

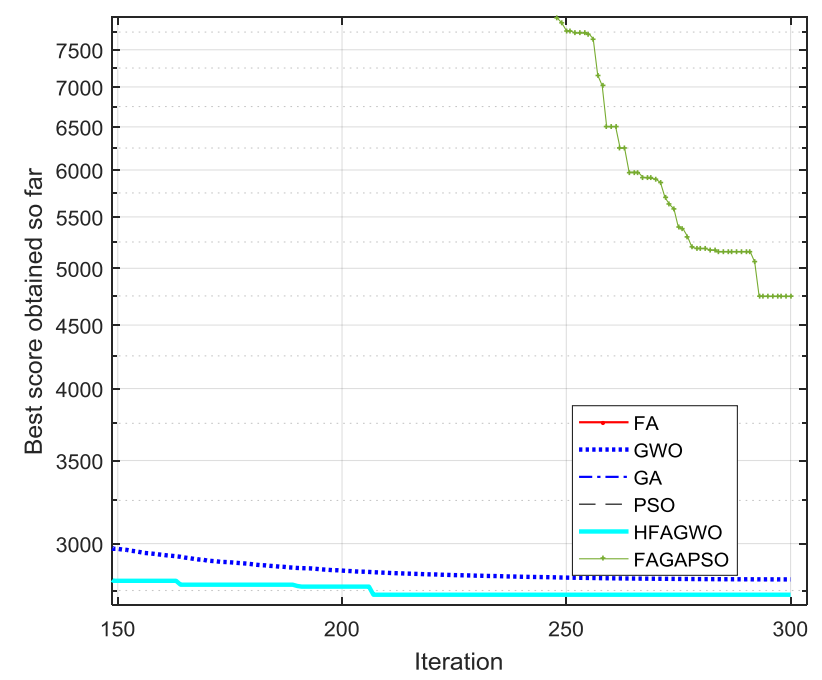

Figure 5: Zoomed in Comparison of Convergence Curve for FAGAPSO, GWO and HFAGWOPSO

The figures show that HFAGWOPSO has the best convergence rate followed by GWO. This is because of firefly movement with PSO operators that is introduced into GWO that improves its exploration ability. HFAGWOPSO has a better objective function value compared to GWO at every iteration. It can be seen that the addition of firefly 
movement with PSO operators into GWO improves the convergence of GWO.

\subsection{Analysis of Performance of HFAGWOPSO}

Power allocation in a TVWS network is a continuous optimization problem. Results have shown that for a continuous optimization problem, HFAGWOPSO is superior to all the algorithms under consideration. Results have shown that HFAGWOPSO outperforms GWO, GA, FA, PSO and FAGAPSO in terms of objective function value, sum power and sum throughput. HFAGWOPSO also has the best convergence rate.

Performance of population based metaheuristic algorithm depends on exploration ability and exploitation ability [10], [11], [23]. There needs to be a sustained exploration in addition exploitation over the course of iterations of the algorithm. Convergence curve in Figures 4 and 5 shows GWO has a better ability to balance between exploration and exploitation and hence it is able to continuously improve objective function value for the entire 300 iterations unlike FA, GA and PSO. FA, GA and PSO converge to a solution which cannot improve after fifteen iterations because they have a poor exploration ability compared to HFAGWOPSO and GWO.

\section{Conclusion}

In this paper, a TVWS network power allocation algorithm based on hybrid grey wolf optimizer and firefly algorithm has been presented. Simulation results show that HFAGWOPSO achieves the best sum throughput, sum power and percentage of SUs less than SU SINR threshold. HFAGWOPSO also has the best convergence rate. Addition of firefly movement into GWO will increase the running time of GWO. Future work analyzing the running time and complexity of the algorithm and find areas of improvement.

\section{References}

[1] K. Patil, R. Prasad, and K. Skouby, "A survey of worldwide spectrum occupancy measurement campaigns for cognitive radio," in International Conference on Devices and Communications (ICDeCom), 2011, pp. 1-5, Accessed: Jun. 25, 2015. [Online]. Available: http://ieeexplore.ieee.org/xpls/abs_all.jsp?arnumber $=5738472$.

[2] M. Mehdawi, N. Riley, K. Paulson, A. Fanan, and M. Ammar, "Spectrum occupancy survey in HULL-UK for cognitive radio applications: measurement \& analysis," Int. J. Sci. Technol. Res., vol. 2(4), no. 4, pp. 231-236, 2013.

[3] R. Kennedy, K. George, O. Vitalice, and W. Okello-Odongo, "TV white spaces in Africa: Trials and role in improving broadband access in Africa," in AFRICON, 2015, 2015, pp. 1-5, Accessed: Nov. 25, 2016. [Online]. Available: http://ieeexplore.ieee.org/xpls/abs_all.jsp?arnumber $=7331920$.

[4] Cristian Gomez, "TV White Spaces: Managing Spaces or Better Managing Inefficiencies," in TV White Spaces: A Pragmatic Approach, P. Pietrosemoli and M. Zennaro, Eds. 2013.

[5] K. Ronoh, G. Kamucha, T. Olwal, and T. Omwansa, "Improved Resource Allocation for TV White Space Network Based on Modified Firefly Algorithm," J. Comput. Inf. Technol., vol. 26(3), no. 3, pp. 167-167, Sep. 2018.

[6] M. Nekovee, T. Irnich, and J. Karlsson, "Worldwide trends in regulation of secondary access to white spaces using cognitive radio," Wirel. Commun. IEEE, vol. 19(4), no. 4, pp. 32-40, 2012.

[7] M. Fadda, V. Popescu, M. Murroni, P. Angueira, and J. Morgade, "On the Feasibility of Unlicensed Communications in the TV White Space: Field Measurements in the UHF Band," Int. J. Digit. Multimed. Broadcast., vol. 2015, pp. 1-8, 2015, doi: 10.1155/2015/319387.

[8] "Comparison of Hybrid Firefly Algorithms for Binary and Continuous Optimization Problems in a TV White Space Network," WSEAS Trans. Commun., vol. 19, Oct. 2020, doi: 10.37394/23204.2020.19.18.

[9] Z. Beheshti and S. M. H. Shamsuddin, "A Review of Population-based Meta-Heuristic Algorithm," Int. J. Adv. Soft Comput. Appl., vol. 5, no. 1, p. 36, 2013.

[10] P. J. Gaidhane and M. J. Nigam, “A hybrid grey wolf optimizer and artificial bee colony algorithm for enhancing the performance of complex systems," J. Comput. Sci., vol. 27(1), pp. 284-302, Jul. 2018, doi: 10.1016/j.jocs.2018.06.008.

[11] N. Singh and S. B. Singh, "Hybrid Algorithm of Particle Swarm Optimization and Grey Wolf Optimizer for Improving Convergence Performance," J. Appl. Math., vol. 2017(1), pp. 115, 2017, doi: 10.1155/2017/2030489.

[12] S. Mirjalili, S. M. Mirjalili, and A. Lewis, "Grey Wolf Optimizer," Adv. Eng. Softw., vol. 69, pp. 46-61, Mar. 2014, doi: 10.1016/j.advengsoft.2013.12.007.

[13] M. Panda and B. Das, "Grey Wolf Optimizer and Its Applications: A Survey," in 
Proceedings of the Third International Conference

on Microelectronics, Computing and Communication Systems, vol. 556, V. Nath and J. K. Mandal, Eds. Singapore: Springer Singapore, 2019, pp. 179-194.

[14] K. Ronoh, G. Kamucha, and T. Omwansa, "Comparison of Hybrid Firefly Algorithms for Power Allocation in a TV White Space Network," Int. J. Comput. Appl., vol. 178, no. 38, pp. 37-43, Aug. 2019, doi: 10.5120/ijca2019919264.

[15] R. Kennedy, K. George, O.-O. William, O. Thomas, and O. Tonny, "Firefly algorithm based power control in wireless TV white space network," in AFRICON, 2017 IEEE, 2017, pp. 155-160.

[16] E. Emary, H. M. Zawbaa, and A. E. Hassanien, "Binary grey wolf optimization approaches for feature selection," Neurocomputing, vol. 172, pp. 371-381, Jan. 2016, doi: 10.1016/j.neucom.2015.06.083.

[17] I. Fister, I. Fister, X.-S. Yang, and J. Brest, "A comprehensive review of firefly algorithms," Swarm Evol. Comput., vol. 13(1), pp. 34-46, Dec. 2013, doi: 10.1016/j.swevo.2013.06.001.

[18] X.-S. Yang, "Firefly algorithms for multimodal optimization," in International Symposium on Stochastic Algorithms, 2009, pp. 169-178, Accessed: Nov. 21, 2016. [Online]. Available:

http://link.springer.com/chapter/10.1007/978-3-64204944-6 14.

[19] $\bar{S}$. Motiian, M. Aghababaie, and $H$. Soltanian-Zadeh, "Particle Swarm Optimization (PSO) of power allocation in cognitive radio systems with interference constraints," in 2011 th IEEE International Conference on Broadband Network and Multimedia Technology, Shenzhen, China, Oct. 2011, pp. 558-562, doi: 10.1109/ICBNMT.2011.6155997.

[20] S. Arunachalam, T. AgnesBhomila, and M. Ramesh Babu, "Hybrid Particle Swarm Optimization Algorithm and Firefly Algorithm Based Combined Economic and Emission Dispatch Including Valve Point Effect," in Swarm, Evolutionary, and Memetic Computing, vol. 8947, B. K. Panigrahi, P. N. Suganthan, and S. Das, Eds. Cham: Springer International Publishing, 2015, pp. 647-660.

[21] P. Kora and K. S. Rama Krishna, "Hybrid firefly and Particle Swarm Optimization algorithm for the detection of Bundle Branch Block," Int. J. Cardiovasc. Acad., vol. 2(1), no. 1, pp. 44-48, Mar. 2016, doi: 10.1016/j.ijcac.2015.12.001.

[22] O. Katircioglu, H. Isel, O. Ceylan, F. Taraktas, and H. B. Yagci, "Comparing ray tracing, free space path loss and logarithmic distance path loss models in success of indoor localization with RSSI," in 2011 19thTelecommunications Forum (TELFOR) Proceedings of Papers, Belgrade, Serbia, Nov. 2011, pp. 313-316, doi: 10.1109/TELFOR.2011.6143552.

[23] X.-S. Yang and X. He, "Firefly Algorithm: Recent Advances and Applications," Int. J. Swarm Intell., vol. 1, no. 1, p. 36, 2013, doi: 10.1504/IJSI.2013.055801.

\section{Contribution of individual authors to the creation of a scientific article (ghostwriting policy)}

Kennedy Ronoh carried out the programming and simulation and also wrote the original draft.

George Kamucha was responsible for the draft review, supervision and validation.

\section{Creative Commons Attribution}

\section{License 4.0 (Attribution 4.0 \\ International , CC BY 4.0)}

This article is published under the terms of the Creative Commons Attribution License 4.0 https://creativecommons.org/licenses/by/4.0/deed.en US 